\title{
Two-Dimensional DOA Estimation for Monostatic MIMO Radar with Electromagnetic Vector Received Sensors
}

\author{
Guimei Zheng $^{1,2}$ and Jun Tang ${ }^{1}$ \\ ${ }^{1}$ Department of Electronic Engineering, Tsinghua University, Beijing 100084, China \\ ${ }^{2}$ Air and Missile Defense College, Air Force Engineering University, Xian 710051, China \\ Correspondence should be addressed to Guimei Zheng; zheng-gm@163.com
}

Received 11 May 2016; Revised 13 July 2016; Accepted 25 July 2016

Academic Editor: Ana Alejos

Copyright (c) 2016 G. Zheng and J. Tang. This is an open access article distributed under the Creative Commons Attribution License, which permits unrestricted use, distribution, and reproduction in any medium, provided the original work is properly cited.

\begin{abstract}
We study two-dimensional direction of arrival (2D-DOA) estimation problem of monostatic MIMO radar with the receiving array which consists of electromagnetic vector sensors (EMVSs). The proposed angle estimation algorithm can be applied to the arbitrary and unknown array configuration, which can be summarized as follows. Firstly, EMVSs in the receiver of a monostatic MIMO radar are used to measure all six electromagnetic-field components of an incident wavefield. The vector sensor array with the six unknown electromagnetic-field components is divided into six spatially identical subarrays. Secondly, ESPRIT is utilized to estimate the rotational invariant factors (RIFs). Parts of the RIFs are picked up to restore the source's electromagnetic-field vector. Last, a vector cross product operation is performed between electric field and magnetic field to obtain the Pointing vector, which can offer the 2D-DOA estimation. Prior knowledge of array elements' positions and angle searching procedure are not necessary for the proposed 2D-DOA estimation method. Simulation results prove the validity of the proposed method.
\end{abstract}

\section{Introduction}

MIMO radar has many merits in target detection and parameter estimation [1]. Therefore, it becomes a hot topic for researchers in the last decade [2-10]. The parameter estimation (such as direction of arrival (DOA) or polarization state angle (PSA)) is one of the main tasks in a radar system. Reference [2] separates the orthogonal signal received in receiving array by using the method of matched filtering to get a virtual array. It has more degrees of freedom of radar system than that of a conventional phased array radar with one version of signal waveform. So it can improve the DOA estimation performance. Reference [3] illuminates that the maximum number of localizable targets of MIMO radar is many times more than that of a counterpart phased array radar. Then, according to the characteristic of MIMO radar, many new parameter estimation algorithms have been developed. Firstly, classic Capon, APES, and CAPES algorithms are applied to monostatic MIMO radar to estimate DOA and amplitude of signals [4]. According to rotational invariance of transmitting and receiving array in bistatic
MIMO radar [5], ESPRIT algorithm is used to estimate direction of departure (DOD) and DOA, but the algorithm needs additional pairing program between DODs and DOAs. So real-valued processing is introduced into bistatic MIMO radar to avoid this problem, and it also can improve estimation performance in the case of low snapshots [6]. In [7], by using the property of Kronecker product, reduceddimensional MUSIC algorithm is presented, which employs one-dimensional angle searching to avoid the high computational cost compared with conventional two-dimensional angle searching algorithm. In addition, alternating projection based maximum likelihood estimation can provide more accurate estimations of target's DOD and DOA [8], which outperforms the existing ESPRIT and MUSIC algorithms. Reference [9] presents a low-complexity algorithm with local one-dimensional searching for joint angle and mutual coupling estimation in bistatic MIMO radar. Reference [10] proposes the Jacobi-Anger expansion to transform the steering vector of uniform circular array into a steering vector with a Vandermonde structure, and then polynomial root finding algorithm is used to estimate DOA and DOD of the targets. 
Combined with the advantages of polarization diversity offered by vector antenna and waveform diversity offered by colocated MIMO radar, Jiang et al. [11] proposes polarimetric MIMO radar. It uses scalar antenna in transmitting array and cross-dipole vector antenna at receiving array. Then ESPRIT algorithm is used to estimate DOD, DOA, and PSA, but the method needs additional pairing program. ESPRIT-Root MUSIC algorithm [12] is extended to polarimetric MIMO radar in order to achieve automatic pairing. In addition, it has low complexity because of the absence of angle searching [13]. Reference [14] introduces parallel factor analysis to polarimetric MIMO radar, which can improve spatial resolution and achieve the goal of three-dimensional positioning. Note that the cross-dipole antennas are used in the above two papers. Therefore, the vector antennas only acquire two-component electric field of source. Reference [15] uses six-component electromagnetic vector sensor (EMVS) [16] to increase the information of a single vector antenna. It generally uses three orthogonal electric dipoles and magnetic loops to measure the three electric-field and three magneticfield parameters of the incident signals, respectively. So there are more advantages in DOA estimation because of using more information. Unfortunately, the method in [15] is only applied to the array configuration with a single received electromagnetic vector sensor.

In this paper, we solve the problem of two-dimensional (2D) DOA estimation of monostatic MIMO radar, whose received array consists of many EMVS antennas. We will show how to find the rotational invariance of spatialpolarimetric domain (SPD) in our signal model. Then a corresponding ESPRIT-like method is proposed to estimate 2D-DOA and PSA in monostatic MIMO radar. The proposed 2D-DOA and PSA estimation method does not need angle searching or any information about array configuration. Simulation results illuminate the advantages of the proposed method. Note that the proposed method can be seen as the extensional version of [17].

Notation. Superscripts $(\cdot)^{*},(\cdot)^{T}$, and $(\cdot)^{H}$ denote complex conjugation, transpose, and conjugate transpose, respectively. $\odot, \times, \otimes$, and $\oplus$ denote Hadamard-Schur product, vector cross product, Kronecker product, and Khatri-Rao product (columnwise Kronecker product), respectively. $\operatorname{Re}\{\cdot\}$ represents the real-value part of the entity inside. $\mathbf{I}_{M N}$ is $M N \times M N$ identity matrix. $\operatorname{Tr}\{\cdot\}$ denotes the trace of the entity inside. $\angle z$ is the angle of complex $z$, which is in $(-\pi, \pi] .\|\cdot\|$ denotes Frobenius norm. $|\cdot|$ denotes absolute value.

\section{Signal Model}

Consider a monostatic MIMO radar; the transmitting array has $M$ antennas. The receiving array is composed of $N$ six-component EMVSs, as shown in Figure 1. Define the location of the $m$ th sensor in the transmitting array as $\left(x_{t m}, y_{t m}, z_{t m}\right), m=1, \ldots, M$ and the location of the $n$th EMVS in the receiving array as $\left(x_{r n}, y_{r n}, z_{r n}\right), n=1, \ldots, N$.

The $M$ transmitting antennas transmit $M$ orthogonal signals, which have the same bandwidth and center frequency; that is, $\mathbf{s}(\tau)=\left[s_{1}(\tau), \ldots, s_{M}(\tau)\right]^{T} \in \mathbb{C}^{M \times 1}$, where $s_{i}(\tau)$ denotes $i$ th transmitted signal; $\tau=1, \ldots, \Gamma$ is the sampling index in fast time; therefore $(1 / \Gamma) \sum_{\tau=1}^{\Gamma} \mathbf{s}(\tau) \mathbf{s}(\tau)^{H}=\mathbf{I}_{M}$. Assume that there are $K$ far-field point targets. The 2D-DOA of target are azimuth angle $\phi \in[0,2 \pi)$ and elevation angle $\theta \in[0, \pi]$, which are measured from positive $x$-axis and positive $z$-axis, respectively. PSA of target can be expressed as auxiliary polarization angle $\gamma \in[0, \pi / 2]$ and polarization phase difference $\eta \in(-\pi, \pi][16]$, respectively. Then received signal can be represented as

$$
\begin{aligned}
\mathbf{x}(t, \tau)= & \mathbf{A}_{r}(\theta, \phi) \\
& \oplus \mathbf{A}_{\mathrm{pol}}(\theta, \phi, \gamma, \eta) \operatorname{diag}[\mathbf{b}(t)] \mathbf{A}_{t}^{T}(\theta, \phi) \mathbf{s}(\tau) \\
& +\mathbf{w}(t, \tau) \in \mathbb{C}^{6 N \times 1},
\end{aligned}
$$

where $t$ denotes slow-time index, namely, snapshot index. $\mathbf{A}_{r}(\theta, \phi)=\left[\mathbf{a}_{r}\left(\theta_{1}, \phi_{1}\right), \ldots, \mathbf{a}_{r}\left(\theta_{K}, \phi_{K}\right)\right] \in \mathbb{C}^{N \times K}$ denotes receiving spatially steering matrix. $\mathbf{A}_{t}(\theta$, $\phi)=\left[\mathbf{a}_{t}\left(\theta_{1}, \phi_{1}\right), \ldots, \mathbf{a}_{t}\left(\theta_{K}, \phi_{K}\right)\right] \in \mathbb{C}^{M \times K}$ denotes transmitting spatially steering matrix. $\mathbf{A}_{\text {pol }}(\theta, \phi, \gamma, \eta)=\left[\mathbf{a}_{\text {pol }}\left(\theta_{1}\right.\right.$, $\left.\left.\phi_{1}, \gamma_{1}, \eta_{1}\right), \ldots, \mathbf{a}_{\text {pol }}\left(\theta_{K}, \phi_{K}, \gamma_{K}, \eta_{K}\right)\right]$ denotes SPD steering matrix of a single collocated EMVS. $\mathbf{a}_{r}\left(\theta_{k}, \phi_{k}\right), \mathbf{a}_{t}\left(\theta_{k}, \phi_{k}\right)$, and $\mathbf{a}_{\text {pol }}\left(\theta_{k}, \phi_{k}, \gamma_{k}, \eta_{k}\right)$ are, respectively, given as follows:

$$
\begin{aligned}
& \mathbf{a}_{r}\left(\theta_{k}, \phi_{k}\right) \\
& =\left[\exp \left(-j \frac{2 \pi}{\lambda}\left(x_{r 1} \sin \theta_{k} \cos \phi_{k}+y_{r 1} \sin \theta_{k} \sin \phi_{k}+z_{r 1} \cos \theta_{k}\right)\right), \ldots, \exp \left(-j \frac{2 \pi}{\lambda}\left(x_{r N} \sin \theta_{k} \cos \phi_{k}+y_{r N} \sin \theta_{k} \sin \phi_{k}+z_{r N} \cos \theta_{k}\right)\right)\right]^{T}, \\
& \mathbf{a}_{t}\left(\theta_{k}, \phi_{k}\right)=\left[\exp \left(-j \frac{2 \pi}{\lambda}\left(x_{t 1} \sin \theta_{k} \cos \phi_{k}+y_{t 1} \sin \theta_{k} \sin \phi_{k}+z_{t 1} \cos \theta_{k}\right)\right), \ldots, \exp \left(-j \frac{2 \pi}{\lambda}\left(x_{t M} \sin \theta_{k} \cos \phi_{k}+y_{t M} \sin \theta_{k} \sin \phi_{k}+z_{t M} \cos \theta_{k}\right)\right)\right]^{T}
\end{aligned}
$$

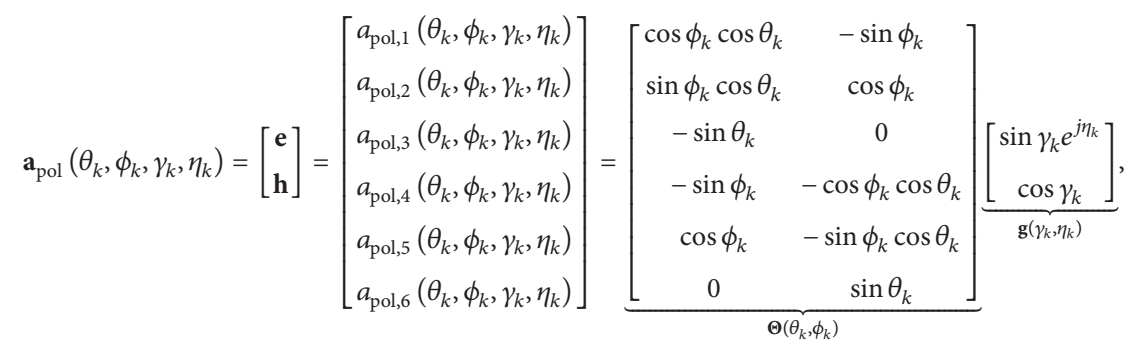




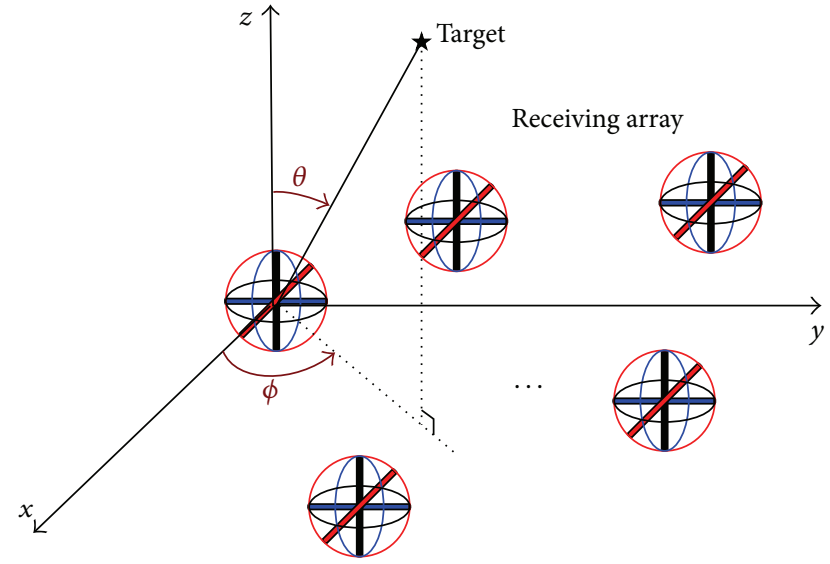

FIgURE 1: Monostatic MIMO radar with arbitrarily spaced electromagnetic vector received sensors at unknown locations.

where $\mathbf{e}$ (the first three elements of $\left.\mathbf{a}_{\text {pol }}\left(\theta_{k}, \phi_{k}, \gamma_{k}, \eta_{k}\right)\right)$ and $\mathbf{h}$ (the last three elements of $\left.\mathbf{a}_{\text {pol }}\left(\theta_{k}, \phi_{k}, \gamma_{k}, \eta_{k}\right)\right)$ denote electricfield vector and magnetic-field vector, respectively. $\mathbf{b}(t)=$ $\left[\gamma_{1}(t), \ldots, \gamma_{K}(t)\right]^{T} \in \mathbb{C}^{K \times 1}$ denotes reflection coefficient vector, where $\gamma_{k}(t)=\alpha_{k} \exp \left(j 2 \pi f_{k} t\right)$. The amplitude of reflection coefficient $\alpha_{k}$ is determined by target's radar cross section (RCS). The phase of reflection coefficient is determined by Doppler frequency of target. $\mathbf{w}(t, \tau) \in \mathbb{C}^{6 N \times 1}$ denotes additive white Gaussian noise with zero mean and covariance matrix $\sigma_{n}^{2} \mathbf{I}_{6 N}$.

Multiply $\mathbf{x}(t, \tau)$ by $(1 / \Gamma) s_{m}(\tau)^{H}, m=1, \ldots, M$; that is, perform matched filtering using the $M$ transmitting signals; the output can be expressed as

$$
\begin{aligned}
& \mathbf{X}(t)=\frac{1}{\Gamma} \sum_{\tau=1}^{\Gamma} \mathbf{x}(t, \tau) \mathbf{s}(\tau)^{H}=\mathbf{A}_{r}(\theta, \phi) \\
& \oplus \mathbf{A}_{\mathrm{pol}}(\theta, \phi, \gamma, \eta) \operatorname{diag}[\mathbf{b}(t)] \mathbf{A}_{t}^{T}(\theta, \phi) \frac{1}{\Gamma} \\
& \quad \cdot \sum_{\tau=1}^{\Gamma} \mathbf{s}(\tau) \mathbf{s}(\tau)^{H}+\mathbf{N}(t)=\mathbf{A}_{r}(\theta, \phi) \\
& \oplus \mathbf{A}_{\mathrm{pol}}(\theta, \phi, \gamma, \eta) \operatorname{diag}[\mathbf{b}(t)] \mathbf{A}_{t}^{T}(\theta, \phi)+\mathbf{N}(t),
\end{aligned}
$$

where $\mathbf{N}(t)=(1 / \Gamma) \sum_{\tau=1}^{\Gamma} \mathbf{w}(t, \tau) \mathbf{s}(\tau)^{H}$. Vectorizing (5) yields the following $6 M N$-dimensional column vector:

$$
\begin{aligned}
y(t) & =\operatorname{vec}\{\mathbf{X}(t)\}=\mathbf{A}(\theta, \phi, \gamma, \eta) \mathbf{b}(t)+\mathbf{n}(t) \\
& \in \mathbb{C}^{6 M N \times 1},
\end{aligned}
$$

where $\mathbf{A}(\theta, \phi, \gamma, \eta)=\mathbf{A}_{t}(\theta, \phi) \oplus \mathbf{A}_{r}(\theta, \phi) \oplus \mathbf{A}_{\text {pol }}(\theta, \phi, \gamma, \eta) \epsilon$ $\mathbb{C}^{6 M N \times K}$, whose columns are defined as virtual array steering vector $\mathbf{a}(\theta, \phi, \gamma, \eta)=\mathbf{a}_{t}(\theta, \phi) \otimes \mathbf{a}_{r}(\theta, \phi) \otimes \mathbf{a}_{\text {pol }}(\theta, \phi, \gamma, \eta)$. And the noise is transformed to $\mathbf{n}(t)=\operatorname{vec}[\mathbf{N}(t)]$. The noise component corresponding to $m$ th transmitting antenna and

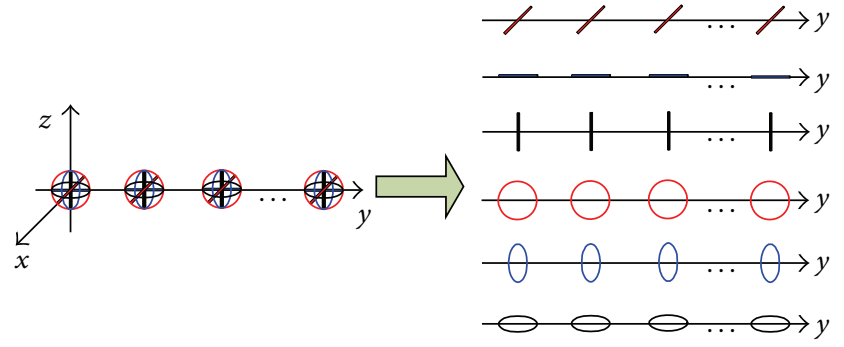

FIGURE 2: Six spatially identical subarrays offered by EMVS array.

the $n$th receiving antenna is $\mathbf{n}_{n, m}=\mathbf{w}_{n} \mathbf{s}_{m}^{H}$. Then the elements of corresponding noise covariance matrix are equal to

$$
\begin{aligned}
& E\left[\mathbf{n}_{n_{2}, m_{2}}^{H} \cdot \mathbf{n}_{n_{1}, m_{1}}\right]=E\left\{\left[\mathbf{w}_{n_{2}} \mathbf{s}_{m_{2}}^{H}\right]^{H} \cdot \mathbf{w}_{n_{1}} \mathbf{s}_{m_{1}}^{H}\right\} \\
& =E\left[\mathbf{s}_{m_{2}} \mathbf{w}_{n_{2}}^{H} \cdot \mathbf{w}_{n_{1}} \mathbf{s}_{m_{1}}^{H}\right] \\
& = \begin{cases}0 & \text { else } \\
\sigma_{n}^{2}, & n_{1}=n_{2}, \quad m_{1}=m_{2} .\end{cases}
\end{aligned}
$$

It implies that $\mathbf{n}(t)$ is still a complex Gaussian white noise with zero mean and covariance matrix $\sigma_{n}^{2} \mathbf{I}_{6 M N}$.

\section{Proposed Algorithm}

For virtual array, after matched filtering, we extract the component with the same orient in EMVSs to consist a subarray. Then we can obtain six spatially identical subarrays. Here we take some EMVSs located in $y$-axis, for example, as shown in Figure 2.

According to the SPD rotational invariance of the six subarrays, we can get $C_{6}^{2}=15$ rotational invariant factors (RIFs) by using ESPRIT algorithm. According to the knowledge of [17], we can calculate the incident electromagnetic wave's electric field and magnetic field by only selecting five RIFs. Then we can perform vector cross product between electric field and magnetic field to obtain the Pointing vector of source. Last, 2D-DOA can be obtained by the triangle operation with the Pointing vector. The detailed derivation is given in the rest of paper.

For $L$ snapshots, covariance matrix of data (6) is calculated by means of maximum likelihood estimation: $\widehat{\mathbf{R}}=$ $1 / L \sum_{t=1}^{L} \mathbf{y}(t) \cdot \mathbf{y}(t)^{H}, t=1, \ldots, L$. Then eigendecomposition is performed with the covariance matrix $\widehat{\mathbf{R}}$ to obtain $\widehat{\mathbf{R}}=\mathbf{E}_{S} \sum_{S} \mathbf{E}_{S}^{H}+\sigma_{n}^{2} \mathbf{E}_{N} \mathbf{E}_{N}^{H}$, where $\mathbf{E}_{S}$ denotes $6 M N \times K$ signal subspace. Without noise, signal subspace and steering vectors $\left\{\mathbf{a}\left(\theta_{k}, \phi_{k}, \gamma_{k}, \eta_{k}\right), k=1, \ldots, K\right\}$ have same space; that is, $\mathbf{E}_{S}=\mathbf{A T}$. $\mathbf{T}$ denotes a nonsingular matrix. In the following, we will show how to estimate the 2D-DOA and PSA according to signal subspace $\mathbf{E}_{S}$ by means of ESPRIT-like algorithm. 
We firstly choose the array manifold of $i$ th subarray by using selection matrix $\mathbf{J}_{i}$. The following relationship equation with the array manifold will hold for $k$ th target:

$$
\begin{aligned}
\mathbf{J}_{i} \mathbf{a} & \left(\theta_{k}, \phi_{k}, \gamma_{k}, \eta_{k}\right) \\
\quad= & a_{\mathrm{pol}, i}\left(\theta_{k}, \phi_{k}, \gamma_{k}, \eta_{k}\right)\left[\mathbf{a}_{t}\left(\theta_{k}, \phi_{k}\right) \otimes \mathbf{a}_{r}\left(\theta_{k}, \phi_{k}\right)\right] .
\end{aligned}
$$

Selection matrix can be expressed as $\mathbf{J}_{i}=\left[\mathbf{I}_{M N} \otimes \mathbf{e}_{i}\right]^{T}$, where vector $\mathbf{e}_{i}=[\underbrace{0, \ldots, 0}_{i-1}, 1, \underbrace{0, \ldots, 0}_{6-i}]^{T}, i=1, \ldots, 6$. According to (8), we can obtain that the following rotational invariance relationship between $i$ th subarray and $j$ th subarray holds:

$$
\begin{aligned}
& {\left[\mathbf{a}_{t}\left(\theta_{k}, \phi_{k}\right) \otimes \mathbf{a}_{r}\left(\theta_{k}, \phi_{k}\right)\right]=\frac{\mathbf{J}_{i} \mathbf{a}\left(\theta_{k}, \phi_{k}, \gamma_{k}, \eta_{k}\right)}{a_{\mathrm{pol}, i}\left(\theta_{k}, \phi_{k}, \gamma_{k}, \eta_{k}\right)}} \\
& \quad=\frac{\mathbf{J}_{j} \mathbf{a}\left(\theta_{k}, \phi_{k}, \gamma_{k}, \eta_{k}\right)}{a_{\mathrm{pol}, j}\left(\theta_{k}, \phi_{k}, \gamma_{k}, \eta_{k}\right)},
\end{aligned}
$$

where $i, j=1, \ldots, 6$. According to (9), we can obtain

$$
\begin{aligned}
& \mathbf{J}_{i} \mathbf{a}\left(\theta_{k}, \phi_{k}, \gamma_{k}, \eta_{k}\right) \\
& \quad=\mathbf{J}_{j} \mathbf{a}\left(\theta_{k}, \phi_{k}, \gamma_{k}, \eta_{k}\right) \frac{a_{\mathrm{pol}, i}\left(\theta_{k}, \phi_{k}, \gamma_{k}, \eta_{k}\right)}{a_{\mathrm{pol}, j}\left(\theta_{k}, \phi_{k}, \gamma_{k}, \eta_{k}\right)} .
\end{aligned}
$$

Consider all of $K$ targets; (10) can be transformed to matrix form:

$$
\mathbf{J}_{i} \mathbf{A}(\theta, \phi, \gamma, \eta)=\mathbf{J}_{j} \mathbf{A}(\theta, \phi, \gamma, \eta) \boldsymbol{\Phi}_{i, j},
$$

where $\boldsymbol{\Phi}_{i, j}=\operatorname{diag}\left[a_{\mathrm{pol}, i}\left(\theta_{1}, \phi_{1}, \gamma_{1}, \eta_{1}\right) / a_{\mathrm{pol}, j}\left(\theta_{1}, \phi_{1}, \gamma_{1}, \eta_{1}\right), \ldots\right.$, $\left.a_{\mathrm{pol}, i}\left(\theta_{K}, \phi_{K}, \gamma_{K}, \eta_{K}\right) / a_{\mathrm{pol}, j}\left(\theta_{K}, \phi_{K}, \gamma_{K}, \eta_{K}\right)\right]^{T}$. For convenient presentation, we define RIF as $\chi_{k}^{i, j} \stackrel{\text { def }}{=} a_{\text {pol, } i}\left(\theta_{k}, \phi_{k}, \gamma_{k}\right.$, $\left.\eta_{k}\right) / a_{\text {pol, } j}\left(\theta_{k}, \phi_{k}, \gamma_{k}, \eta_{k}\right)$. Substituting $\mathbf{E}_{S}=\mathbf{A T}$ into (11), we can obtain rotational invariant equation about signal subspace $\mathbf{E}_{S}$ :

$$
\mathbf{J}_{i} \mathbf{E}_{S}=\mathbf{J}_{j} \mathbf{E}_{S} \Psi_{i, j}
$$

where $\boldsymbol{\Psi}_{i, j}=\left(\mathbf{T}_{i, j}\right)^{-1} \boldsymbol{\Phi}_{i, j} \mathbf{T}_{i, j}$. In noise scenario, (12) can be solved by least squares or total least squares (TLS) method to obtain $\Psi_{i, j}$. Then, performing eigendecomposition with $\Psi_{i, j}$, RIF $\chi_{k}^{i, j}$ estimation can be obtained.

Note that the RIFs $\chi_{k}^{i, j} \mid i, j=1, \ldots, 6$ in different $\Phi_{i, j}$ have different orders. But there is no mismatch between $\chi_{k}^{i, j}$ and its corresponding eigenvector. Thus, different $\chi_{k}^{i, j}$ can be paired for the same source by pairing the orthogonal rows of $\mathbf{T}_{i, j}$. Take, for example, $\chi_{k}^{2,1}$ and $\chi_{k}^{3,2}$. Let $k$ denote the row index of the matrix element with the largest absolute value in the $f$ th column of the matrix $\left\{\mathbf{T}_{2,1} \cdot\left(\mathbf{T}_{3,2}\right)^{-1}\right\}$. Then the $k$ th row of $\mathbf{T}_{2,1}$ must correspond to the $f$ th row of $\mathbf{T}_{3,2}$. Now, $\left\{\chi_{k}^{2,1}, k=\right.$ $1, \ldots, K\}$ and $\left\{\chi_{k}^{3,2}, k=1, \ldots, K\right\}$ have been correctly paired. All the RIFs $\chi_{k}^{i, j} \mid i, j=1, \ldots, 6$ can be paired by performing the similar processing.
There are $C_{6}^{2}=15$ choices for RIFs $\chi_{k}^{i, j}$ because of $i, j=$ $1, \ldots, 6$. We set $i=j+1$; that is, 5 RIFs $\chi_{k}^{j+1, j}, j=1, \ldots, 5$, are selected. In the following, we will show how to use the five RIFs to calculate the Pointing vector. According to the definition of $\chi_{k}^{i, j}$, the following equations hold:

$$
\begin{aligned}
a_{\mathrm{pol}, 2}\left(\theta_{k}, \phi_{k}, \gamma_{k}, \eta_{k}\right)= & a_{\mathrm{pol}, 1}\left(\theta_{k}, \phi_{k}, \gamma_{k}, \eta_{k}\right) \cdot \chi_{k}^{2,1}, \\
a_{\mathrm{pol}, 3}\left(\theta_{k}, \phi_{k}, \gamma_{k}, \eta_{k}\right)= & a_{\mathrm{pol}, 1}\left(\theta_{k}, \phi_{k}, \gamma_{k}, \eta_{k}\right) \cdot \chi_{k}^{2,1} \cdot \chi_{k}^{3,2}, \\
a_{\mathrm{pol}, 4}\left(\theta_{k}, \phi_{k}, \gamma_{k}, \eta_{k}\right)= & a_{\mathrm{pol}, 1}\left(\theta_{k}, \phi_{k}, \gamma_{k}, \eta_{k}\right) \cdot \chi_{k}^{2,1} \cdot \chi_{k}^{3,2} \\
& \cdot \chi_{k}^{4,3}, \\
a_{\mathrm{pol}, 5}\left(\theta_{k}, \phi_{k}, \gamma_{k}, \eta_{k}\right)= & a_{\mathrm{pol}, 1}\left(\theta_{k}, \phi_{k}, \gamma_{k}, \eta_{k}\right) \cdot \chi_{k}^{2,1} \cdot \chi_{k}^{3,2} \\
& \cdot \chi_{k}^{4,3} \cdot \chi_{k}^{5,4}, \\
a_{\mathrm{pol}, 6}\left(\theta_{k}, \phi_{k}, \gamma_{k}, \eta_{k}\right)= & a_{\mathrm{pol}, 1}\left(\theta_{k}, \phi_{k}, \gamma_{k}, \eta_{k}\right) \cdot \chi_{k}^{2,1} \cdot \chi_{k}^{3,2} \\
& \cdot \chi_{k}^{4,3} \cdot \chi_{k}^{5,4} \cdot \chi_{k}^{6,5} \cdot
\end{aligned}
$$

According to Maxwell equation, we know that the result of cross-multiplication between the electric-field vector and the magnetic-field vector is equal to the Pointing vector as follows:

$$
\begin{aligned}
& {\left[\begin{array}{c}
a_{\mathrm{pol}, 1}\left(\theta_{k}, \phi_{k}, \gamma_{k}, \eta_{k}\right) \\
a_{\mathrm{pol}, 2}\left(\theta_{k}, \phi_{k}, \gamma_{k}, \eta_{k}\right) \\
a_{\mathrm{pol}, 3}\left(\theta_{k}, \phi_{k}, \gamma_{k}, \eta_{k}\right)
\end{array}\right] \times\left[\begin{array}{c}
a_{\mathrm{pol}, 4}\left(\theta_{k}, \phi_{k}, \gamma_{k}, \eta_{k}\right) \\
a_{\mathrm{pol}, 5}\left(\theta_{k}, \phi_{k}, \gamma_{k}, \eta_{k}\right) \\
a_{\mathrm{pol}, 6}\left(\theta_{k}, \phi_{k}, \gamma_{k}, \eta_{k}\right)
\end{array}\right]} \\
& \quad=\mathbf{e} \times \mathbf{h}=\Gamma .
\end{aligned}
$$

Consider $\Gamma \stackrel{\text { def }}{=}\left[\begin{array}{lll}u & v & w\end{array}\right]$ is the Pointing vector, where $u=\sin \theta \cos \phi, v=\sin \theta \sin \phi$, and $w=\cos \theta$ are the three direction cosines aligning to $x$-axis, $y$-axis, and $z$-axis, respectively. Substituting (13) into (14), we have

$$
\begin{gathered}
\left\|a_{\mathrm{pol}, 1}\left(\theta_{k}, \phi_{k}, \gamma_{k}, \eta_{k}\right)\right\|^{2}\left[\begin{array}{c}
1 \\
\chi_{k}^{2,1} \\
\chi_{k}^{2,1} \cdot \chi_{k}^{3,2}
\end{array}\right] \\
\times\left[\begin{array}{c}
\chi_{k}^{2,1} \cdot \chi_{k}^{3,2} \cdot \chi_{k}^{4,3} \\
\chi_{k}^{2,1} \cdot \chi_{k}^{3,2} \cdot \chi_{k}^{4,3} \cdot \chi_{k}^{5,4} \\
\chi_{k}^{2,1} \cdot \chi_{k}^{3,2} \cdot \chi_{k}^{4,3} \cdot \chi_{k}^{5,4} \cdot \chi_{k}^{6,5}
\end{array}\right]=\left[\begin{array}{c}
u \\
v \\
w
\end{array}\right] .
\end{gathered}
$$

We can compute the vector cross product operation of (15) according to the paired estimations $\chi_{k}^{j+1, j}, j=1, \ldots, 5$. Then the estimations of direction cosines $\widehat{u}_{k}$, $\widehat{v}_{k}$, and $\widehat{w}_{k}$ can be obtained by normalized processing with the result of vector 
cross product. Last, azimuth and elevation angle estimations can be obtained by the following triangle operations:

$$
\begin{aligned}
& \widehat{\phi}_{k}=\arctan \left(\frac{\widehat{v}_{k}}{\widehat{u}_{k}}\right), \quad \text { if } \widehat{u}_{k} \geq 0, \\
& \widehat{\phi}_{k}=\arctan \left(\frac{\widehat{v}_{k}}{\widehat{u}_{k}}\right)+\pi, \quad \text { if } \widehat{u}_{k}<0, \\
& \widehat{\theta}_{k}=\arcsin \left(\sqrt{\widehat{u}_{k}^{2}+\widehat{v}_{k}^{2}}\right), \quad \text { if } \widehat{w}_{k} \geq 0, \\
& \widehat{\theta}_{k}=-\arcsin \left(\sqrt{\widehat{u}_{k}^{2}+\widehat{v}_{k}^{2}}\right)+\pi, \quad \text { if } \widehat{w}_{k}<0, \\
& \quad k=1, \ldots, K .
\end{aligned}
$$

From the above processing, we can see that the information of antennas' locations $\left(x_{t m}, y_{t m}, z_{t m}\right), m=1, \ldots, M$, and $\left(x_{r n}, y_{r n}, z_{r n}\right), n=1, \ldots, N$, is not utilized. It can be seen from (4) that

$$
\begin{aligned}
\mathbf{g}\left(\gamma_{k}, \eta_{k}\right)= & {\left[\boldsymbol{\Theta}^{H}\left(\theta_{k}, \phi_{k}\right) \boldsymbol{\Theta}\left(\theta_{k}, \phi_{k}\right)\right]^{-1} \boldsymbol{\Theta}\left(\theta_{k}, \phi_{k}\right)^{H} } \\
& \cdot \widehat{\mathbf{a}}_{\text {pol }}\left(\theta_{k}, \phi_{k}, \gamma_{k}, \eta_{k}\right) .
\end{aligned}
$$

Substituting $\left(\widehat{\theta}_{k}, \widehat{\phi}_{k}\right)$ into (17), we can obtain the estimations of $\mathbf{g}\left(\gamma_{k}, \eta_{k}\right)$. Then we can calculate the corresponding PSA by the following formula:

$$
\begin{aligned}
& \widehat{\gamma}_{k}=\arctan \left|\frac{\left[\widehat{\mathbf{g}}\left(\gamma_{k}, \eta_{k}\right)\right]_{1}}{\left[\widehat{\mathbf{g}}\left(\gamma_{k}, \eta_{k}\right)\right]_{2}}\right| \\
& \widehat{\eta}_{k}=\angle\left[\widehat{\mathbf{g}}\left(\gamma_{k}, \eta_{k}\right)\right]_{1}-\angle\left[\widehat{\mathbf{g}}\left(\gamma_{k}, \eta_{k}\right)\right]_{2} .
\end{aligned}
$$

It can be seen from the above analysis that the proposed algorithm has the following advantages:

(1) Because we do not use the information of array configuration, it can be suitable for arbitrary array.

(2) It requires no knowledge of the locations of antennas.

(3) It has low complexity, since it requires no angle searching.

(4) It has whole sphere validity region for $2 \mathrm{D}-\mathrm{DOA}$ estimation.

(5) It can be applied to EMVSs array in received side, but the method in [15] is only applied to a single EMVS in received side.

Based on the above theoretical analysis, the implementation of the 2D-DOA estimation for monostatic MIMO radar with electromagnetic vector received sensors is summarized as follows.

Step 1. Perform matched filtering processing by using (5) and vectorization by using (6) according to the received data of (1).

Step 2. Compute the estimation of virtual array covariance matrix $\widehat{\mathbf{R}}$ according to received data of (6). Compute the eigenvectors of $\widehat{\mathbf{R}}$ to form the signal subspace $\mathbf{E}_{S}$.
Step 3. Form the spatially rotational invariant equation of (12) according to the signal subspace $\mathbf{E}_{S}$. Calculate the solution of (12) by using TLS, that is, estimation of $\left\{\Psi_{i, j}\right\}$, and then compute the eigenvalues of $\left\{\Psi_{i, j}\right\}$ to obtain the RIFs estimations $\chi_{k}^{j+1, j}, j=1, \ldots, 5$.

Step 4. Complete the pairing of the estimations $\left\{\chi_{k}^{2,1}\right.$, $\left.\chi_{k}^{3,2}, \chi_{k}^{4,3}, \chi_{k}^{5,4}, \chi_{k}^{6,5},(k=1, \ldots, K)\right\}$ for the same incident signal by using the relationship of eigenvalues and their corresponding eigenvectors.

Step 5. Perform the vector cross product operation of (15) according to the paired estimations $\left\{\chi_{k}^{2,1}\right.$, $\left.\chi_{k}^{3,2}, \chi_{k}^{4,3}, \chi_{k}^{5,4}, \chi_{k}^{6,5},(k=1, \ldots, K)\right\}$. Then perform normalized processing to obtain estimations of direction cosines $\widehat{u}_{k}, \widehat{v}_{k}$, and $\widehat{w}_{k}$.

Step 6. Last, we can obtain azimuth and elevation angle estimations by (16). If necessary, we can calculate auxiliary polarization angle and polarization phase difference according to (18).

\section{Complexity Analysis and Maximum Number of Localizable Targets}

The computational complexity of the proposed algorithm is dominated by (1) formation of the covariance matrix of virtual MIMO array radar, (2) calculation of the estimation of array covariance matrix and its eigendecomposition to obtain signal subspace, (3) calculation of five RIFs, and (4) pairing scheme for the five RIFs. Process (1) requires $\mathcal{O}[6 M N \Gamma]$ flops; process (2) requires $\mathcal{O}\left[L(6 M N)^{2}\right]$ flops; process (3) requires $\mathscr{O}\left[(6 M N)^{3}\right]$ flops; process $(4)$ requires $\left.\mathcal{O}\left[5 K^{2} M N\right)\right]$ flops; process (5) requires $\mathcal{O}\left[5 K^{3}\right]$ flops. Therefore, the computational load of proposed algorithm is mainly $\mathcal{O}[6 M N \Gamma+$ $\left.L(6 M N)^{2}+(6 M N)^{3}+5 K^{2} M N\right)+5 K^{3}$ ] flops.

The maximum number of localizable targets of the proposed algorithm is dominated by the choice of subarrays for ESPRIT algorithm. According to the choice of subarrays for ESPRIT in Figure 2, we can obtain that the maximum number of targets that can be localized is $M N$.

\section{Computer Simulations}

In this section, several numerical examples are presented to verify the effectiveness of the proposed algorithm. The root mean squared error (RMSE) of 2D-DOA and PSA estimations is defined as RMSE $=\sqrt{(1 / K) \sum_{k=1}^{K} E\left(\widehat{\rho}_{k}-\rho_{k}\right)^{2}}, \rho=$ $\theta, \phi, \gamma, \eta$. In following simulations, we consider a MIMO radar system with $M=6$ and $N=6$. We set the transmitting antennas located at $x$-axis with half-wavelength spacing and the receiving antennas located in $y$-axis with half-wavelength spacing. So the MIMO radar consists of L-shape array. Note that you can set MIMO radar with arbitrary configuration. There exists $K=2$ uncorrelated targets, which are located at the angles $\left(\theta_{1}, \phi_{1}\right)=$ $\left(30^{\circ}, 40^{\circ}\right),\left(\theta_{2}, \phi_{2}\right)=\left(60^{\circ}, 70^{\circ}\right)$. The incident targets' PSA are 

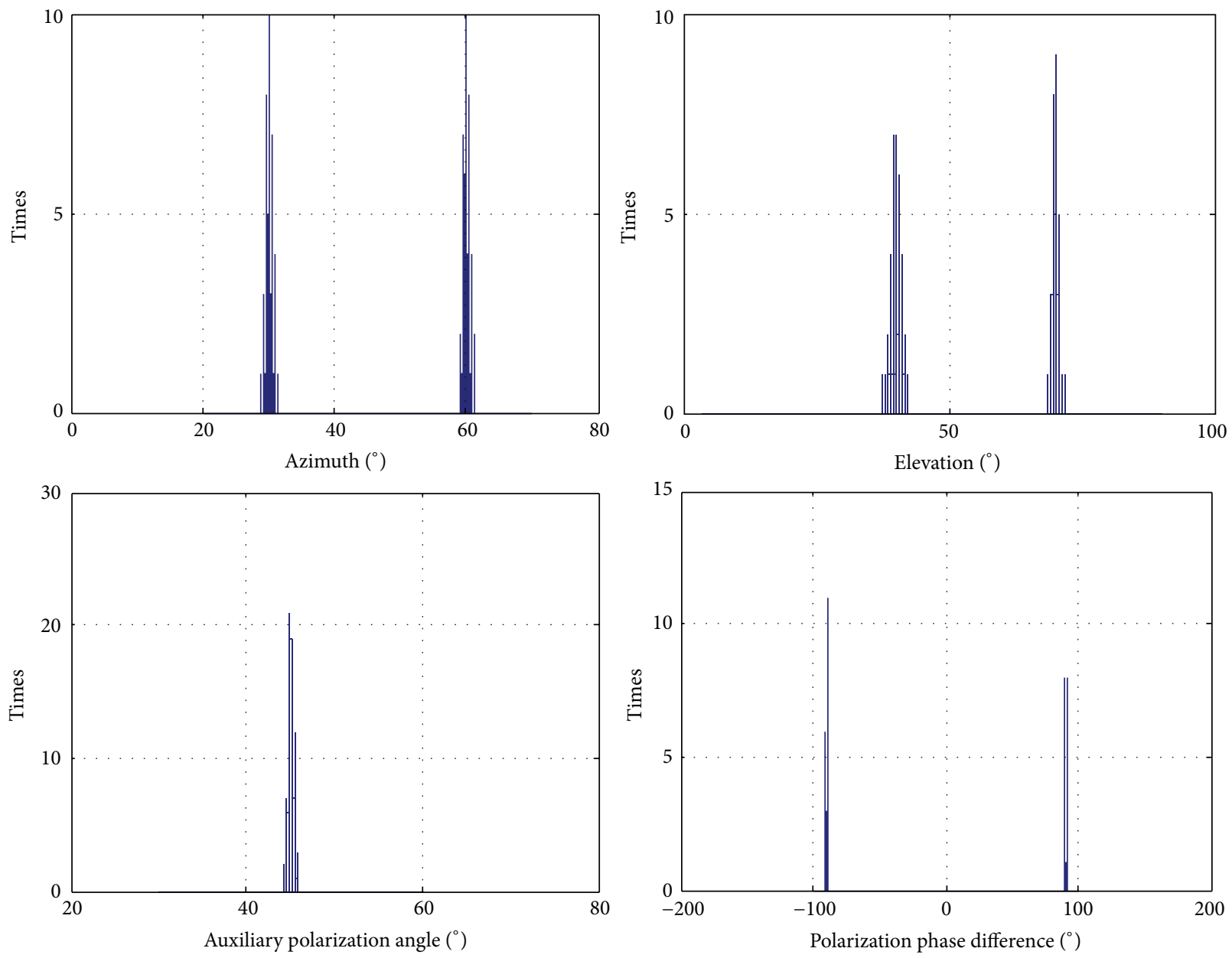

FIGURE 3: The estimation results of two targets with 500 trials.

$\left(\gamma_{1}, \eta_{1}\right)=\left(45^{\circ}, 90^{\circ}\right),\left(\gamma_{2}, \eta_{2}\right)=\left(45^{\circ},-90^{\circ}\right)$. The first target is left-circularly polarized, whereas the second target is rightcircularly polarized. Note that the two targets have identical auxiliary polarization angles.

Figure 3 shows the histograms of estimation results with 500 Monte Carlo trials. In this simulation, assume that SNR = $5 \mathrm{~dB}$ and the number of snapshots is 100 . As can be seen in the figure, the 2D-DOA of the two targets are well localized and the PSA of the two targets are also well estimated.

Figure 4 shows the RMSE of 2D-DOA and PSA versus SNR, compared with CRB. In this simulation, we set $L=200$ and perform 1000 Monte Carlo trials. It is indicated in Figures $4(\mathrm{a})-4(\mathrm{~d})$ that the performance of 2D-DOA estimation of the proposed algorithm becomes better with SNR increasing and there is a gap between proposed algorithm and CRB. We can also see that the performance of PSA estimations of the proposed algorithm is close to CRB. It is well known that DOA estimation performance is critically dependent on array aperture. Large array aperture can offer very accurate estimation. This result occurs because the proposed method does not make use of array aperture but only uses the information inside of the vector sensor. The polarization estimation performance is only dependent on the information inside of the vector sensor [17] and we fully make use of it.
Figure 5 shows the RMSE of 2D-DOA and PSA versus the number of snapshots, compared with CRB. In this simulation, we set SNR $=20 \mathrm{~dB}$ and the other simulation conditions are the same as the above simulation ones. It is indicated in Figures 5(a)-5(d) that the performance of 2DDOA estimation of the proposed algorithm becomes better in collaboration with $L$ increasing and there is a gap between proposed algorithm and CRB. And the performance of PSA estimation of the proposed algorithm is close to CRB. The reason is the same as the above simulation.

\section{Conclusion}

This paper has studied the 2D-DOA estimation problem for monostatic MIMO radar with arbitrary array. ESPRIT-like algorithm is used to calculate RIFs between the six subarrays within six-component EMVS. Then the electric-field and magnetic-field vectors are restored according to the five RIFs. Last, the 2D-DOA is obtained by the Pointing vector offered by the vector cross product between the electric-field and magnetic-field vectors. The proposed algorithm can be suitable for arbitrary array with electromagnetic vector received sensors at unknown locations. This advantage alleviates the nontrivial problem of spatial calibration. Moreover, it has low 


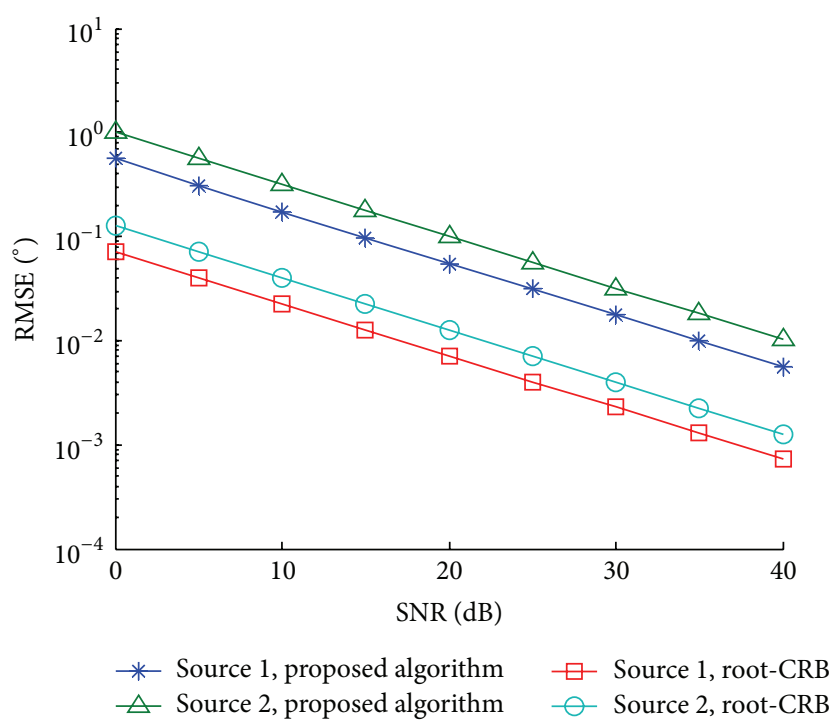

(a) Azimuth

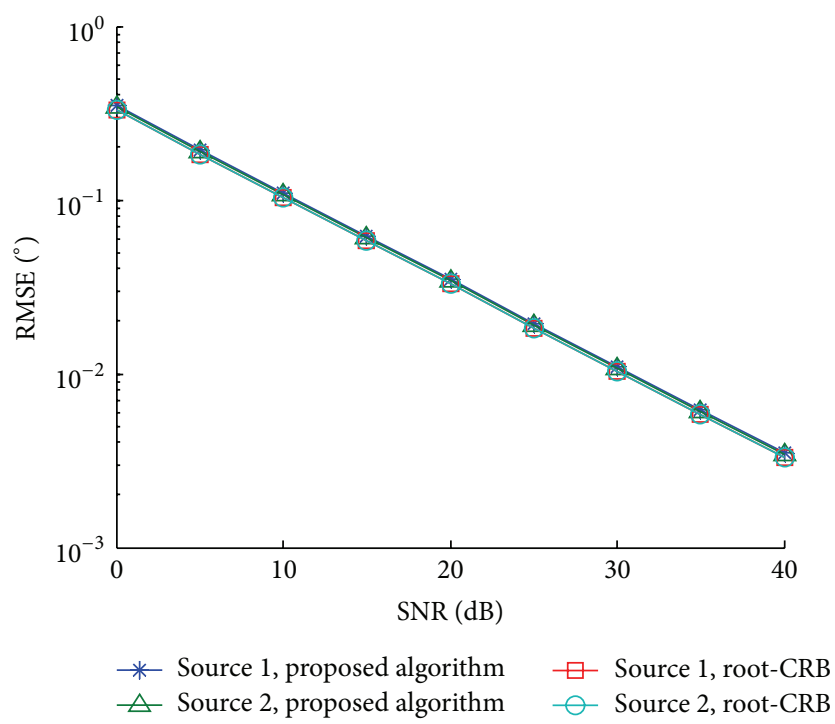

(c) Auxiliary polarization angle

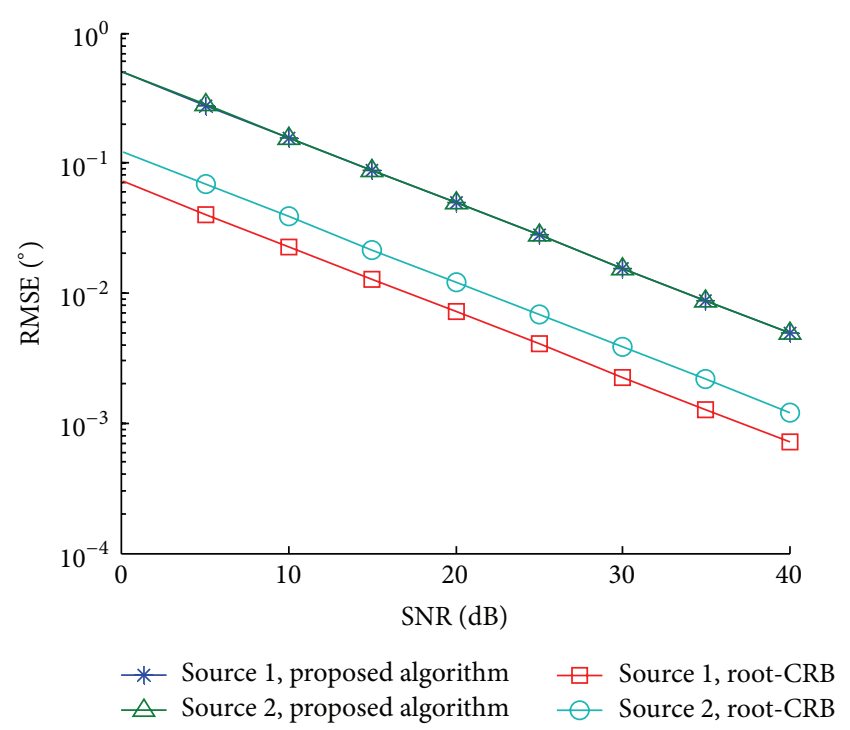

(b) Elevation

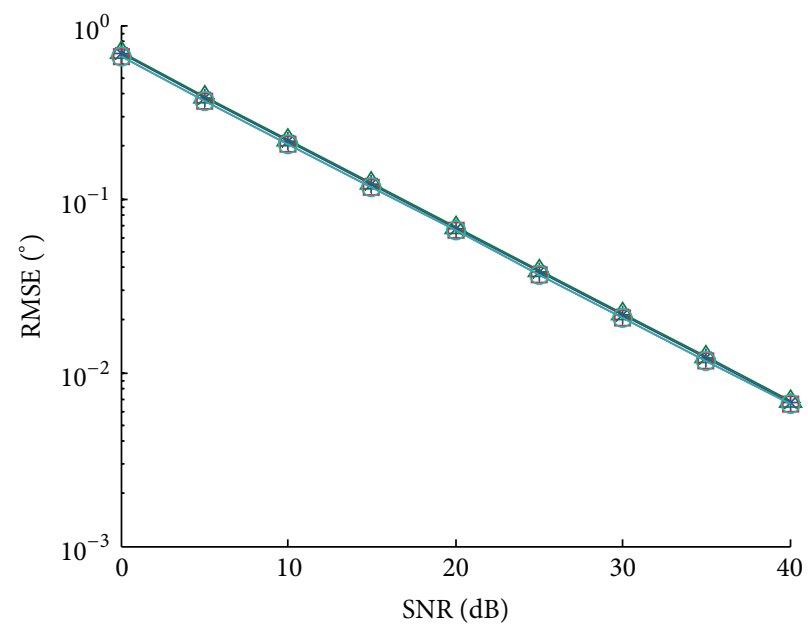

$\begin{aligned} & \text { * Source 1, proposed algorithm } \quad \square \text { Source 1, root-CRB } \\ & \triangle \text { Source 2, proposed algorithm }\end{aligned}-$ Source 2, root-CRB

(d) Polarization phase difference

FIGURE 4: RMSE versus SNR.

complexity because of no angle searching and the 2D-DOA estimation validity region is whole sphere.

\section{Appendix}

The method of derivation of Cramér-Rao Bound (CRB) in $[18,19]$ is used in our signal model. We rewrite $\left\{\theta_{k}, \phi_{k}, \gamma_{k}, \eta_{k} \mid\right.$ $k=1, \ldots, K\}$ as compact vector form:

$$
\begin{aligned}
\boldsymbol{\theta} & =\left[\theta_{1}, \ldots, \theta_{K}\right], \\
\boldsymbol{\psi} & =\left[\phi_{1}, \ldots, \phi_{K}\right], \\
\boldsymbol{\gamma} & =\left[\gamma_{1}, \ldots, \gamma_{K}\right], \\
\boldsymbol{\eta} & =\left[\eta_{1}, \ldots, \eta_{K}\right] .
\end{aligned}
$$

We must firstly compute Fisher information matrix (FIM), which can be expressed as

$$
\mathbf{J}=\left[\begin{array}{llll}
\mathbf{J}_{\theta \theta} & \mathbf{J}_{\theta \psi} & \mathbf{J}_{\theta \gamma} & \mathbf{J}_{\theta \eta} \\
\mathbf{J}_{\psi \theta} & \mathbf{J}_{\psi \psi} & \mathbf{J}_{\psi \gamma} & \mathbf{J}_{\psi \eta} \\
\mathbf{J}_{\gamma \theta} & \mathbf{J}_{\gamma \psi} & \mathbf{J}_{\gamma \gamma} & \mathbf{J}_{\gamma \eta} \\
\mathbf{J}_{\eta \theta} & \mathbf{J}_{\eta \psi} & \mathbf{J}_{\eta \gamma} & \mathbf{J}_{\eta \eta}
\end{array}\right]_{4 K \times 4 K}
$$

According to [20], $(i, j)$ elements of $\mathbf{J}_{\mathbf{h k}},(\mathbf{h}, \mathbf{k}=\boldsymbol{\theta}, \boldsymbol{\psi}, \boldsymbol{\gamma}, \boldsymbol{\eta})$ for $L$ snapshots are equal to

$$
\mathbf{J}_{\mathbf{h k}}(i, j)=L \cdot \operatorname{Tr}\left\{\mathbf{R}^{-1} \frac{\partial \mathbf{R}}{\partial \mathbf{h}_{i}} \mathbf{R}^{-1} \frac{\partial \mathbf{R}}{\partial \mathbf{k}_{j}}\right\},
$$




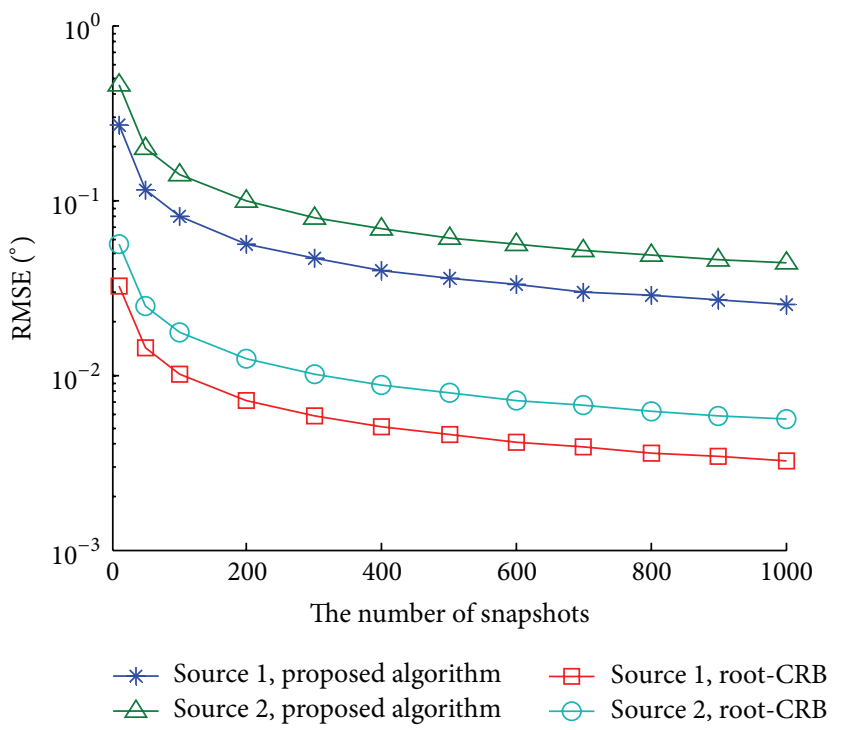

(a) Azimuth

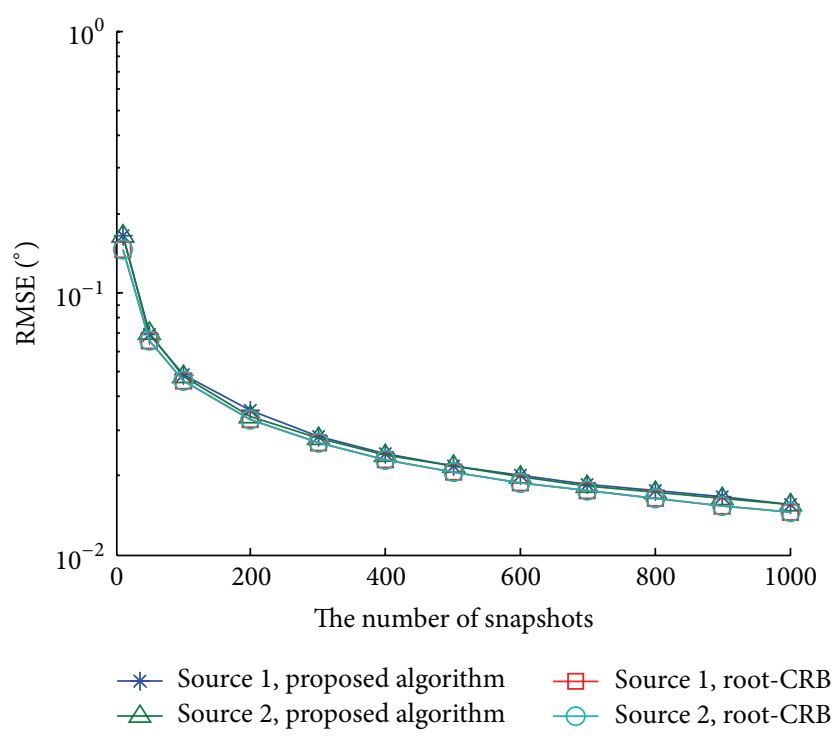

(c) Auxiliary polarization angle

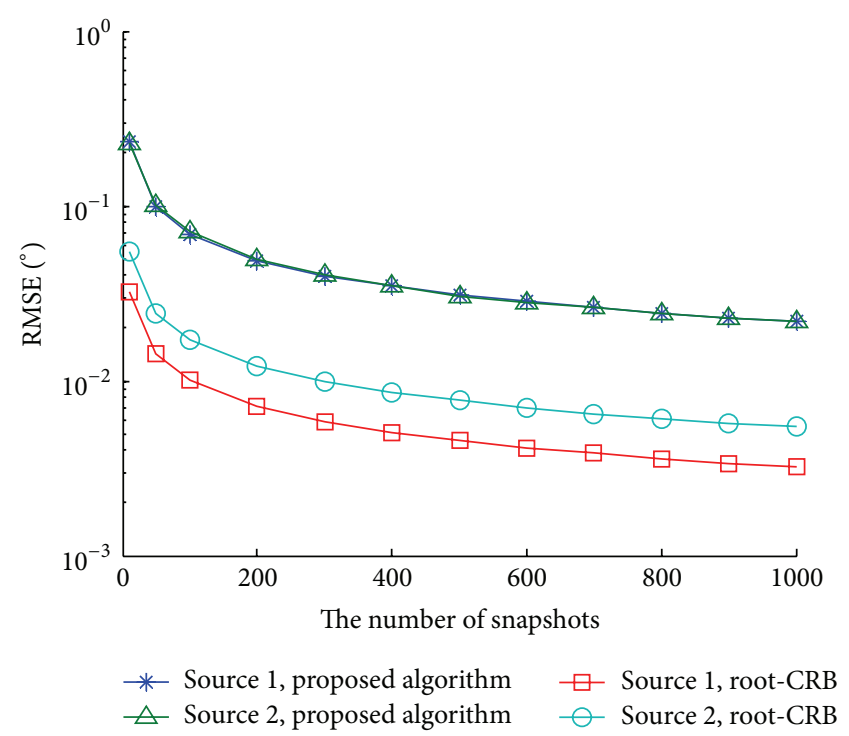

(b) Elevation

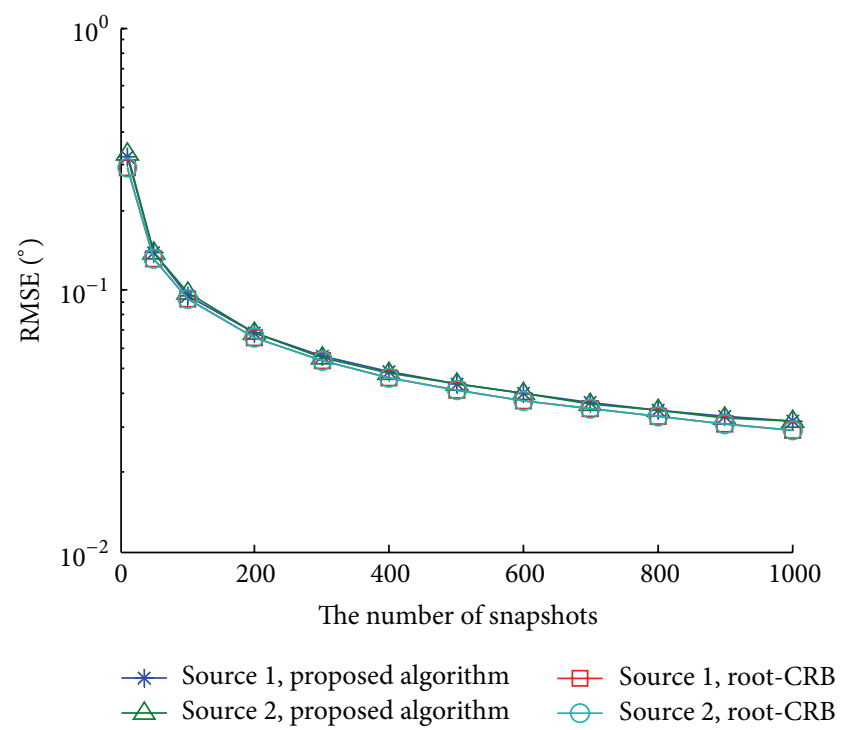

(d) Polarization phase difference

FIGURE 5: RMSE versus number of snapshots.

where $\mathbf{R}$ denotes virtual array covariance matrix. Assume that the incident signals are uncorrelated. For simplicity, $\mathbf{a}_{t}\left(\theta_{k}, \phi_{k}\right), \mathbf{a}_{r}\left(\theta_{k}, \phi_{k}\right), \mathbf{a}_{\mathrm{pol}}\left(\theta_{k}, \phi_{k}, \gamma_{k}, \eta_{k}\right)$, and $\mathbf{a}\left(\theta_{k}, \phi_{k}, \gamma_{k}, \eta_{k}\right)$ are marked with $\mathbf{a}_{t, k}, \mathbf{a}_{r, k}, \mathbf{a}_{\mathrm{pol}, k}$, and $\mathbf{a}_{k}$, respectively. Then the array covariance matrix $\mathbf{R}$ can be unwrapped as

$$
\begin{aligned}
\mathbf{R}= & \sum_{k=1}^{K} \sigma_{s_{k}}^{2}\left[\mathbf{a}_{t, k} \otimes \mathbf{a}_{r, k} \otimes \mathbf{a}_{\mathrm{pol}, k}\right]\left[\mathbf{a}_{t, k} \otimes \mathbf{a}_{r, k} \otimes \mathbf{a}_{\mathrm{pol}, k}\right]^{H} \\
& +\sigma_{n}^{2} \mathbf{I}_{6 M N},
\end{aligned}
$$

where $\sigma_{s_{k}}^{2}$ is the power of $k$ th incident signal. Then the firstorder partial derivatives of $\mathbf{R}$ with respect to $\theta_{k}, \phi_{k}, \gamma_{k}$, and $\eta_{k}$ are equal to

$$
\frac{\partial \mathbf{R}}{\partial \xi_{k}}=\frac{\sigma_{s_{k}}^{2} \partial \mathbf{a}_{k} \mathbf{a}_{k}^{H}}{\partial \xi_{k}}=\sigma_{s_{k}}^{2} \frac{\partial \mathbf{a}_{k}}{\partial \xi_{k}} \mathbf{a}_{k}^{H}+\sigma_{s_{k}}^{2} \mathbf{a}_{k} \frac{\partial \mathbf{a}_{k}^{H}}{\partial \xi_{k}}
$$

where $\xi$ denotes $\theta, \phi, \gamma$, or $\eta$. The results of (A.5) are equal to

$$
\begin{aligned}
\overline{\mathbf{b}}_{\theta_{k}} \stackrel{\text { def }}{=} & \frac{\partial \mathbf{a}_{k}}{\partial \theta_{k}} \\
= & {\left[\mathbf{c}_{1} \odot \mathbf{a}_{t, k}\right] \otimes \mathbf{a}_{r, k} \otimes \mathbf{a}_{\mathrm{pol}, k}+\mathbf{a}_{t, k} \otimes\left[\mathbf{c}_{2} \odot \mathbf{a}_{r, k}\right] } \\
& \otimes \mathbf{a}_{\mathrm{pol}, k}+\mathbf{a}_{t, k} \otimes \mathbf{a}_{r, k} \otimes\left[\mathbf{C}_{3} \mathbf{g}\left(\gamma_{k}, \eta_{k}\right)\right],
\end{aligned}
$$




$$
\begin{aligned}
\overline{\mathbf{b}}_{\phi_{k}} \stackrel{\text { def }}{=} \frac{\partial \mathbf{a}_{k}}{\partial \phi_{k}} & \overline{\mathbf{b}}_{\gamma_{k}} \stackrel{\text { def }}{=} \frac{\partial \mathbf{a}_{k}}{\partial \gamma_{k}}=\mathbf{a}_{t, k} \otimes \mathbf{a}_{r, k} \otimes\left[\Theta\left(\theta_{k}, \phi_{k}\right) \mathbf{c}_{7}\right], \\
=\left[\mathbf{c}_{4} \odot \mathbf{a}_{t, k}\right] \otimes \mathbf{a}_{r, k} \otimes \mathbf{a}_{\mathrm{pol}, k}+\mathbf{a}_{t, k} \otimes\left[\mathbf{c}_{5} \odot \mathbf{a}_{r, k}\right] & \overline{\mathbf{b}}_{\eta_{k}} \stackrel{\text { def }}{=} \frac{\partial \mathbf{a}_{k}}{\partial \eta_{k}}=\mathbf{a}_{t, k} \otimes \mathbf{a}_{r, k} \otimes\left[\Theta\left(\theta_{k}, \phi_{k}\right) \mathbf{c}_{8}\right],
\end{aligned}
$$

where

$$
\begin{aligned}
& \mathrm{c}_{1} \\
& =-j \frac{2 \pi}{\lambda}\left[\left(x_{t 1} \cos \theta_{k} \cos \phi_{k}+y_{t 1} \cos \theta_{k} \sin \phi_{k}-z_{t 1} \sin \theta_{k}\right), \ldots,\left(x_{t M} \cos \theta_{k} \cos \phi_{k}+y_{t M} \cos \theta_{k} \sin \phi_{k}-z_{t M} \sin \theta_{k}\right)\right]^{T}, \\
& \mathrm{c}_{2} \\
& =-j \frac{2 \pi}{\lambda}\left[\left(x_{r 1} \cos \theta_{k} \cos \phi_{k}+y_{r 1} \cos \theta_{k} \sin \phi_{k}-z_{r 1} \sin \theta_{k}\right), \ldots,\left(x_{r N} \cos \theta_{k} \cos \phi_{k}+y_{r N} \cos \theta_{k} \sin \phi_{k}-z_{r N} \sin \theta_{k}\right)\right]^{T}, \\
& \mathbf{C}_{3}=\left[\begin{array}{cccccc}
-u_{k} & -v_{k} & -w_{k} & 0 & 0 & 0 \\
0 & 0 & 0 & u_{k} & v_{k} & w_{k}
\end{array}\right]^{T} \\
& \mathbf{c}_{4}=-j \frac{2 \pi}{\lambda}\left[\left(-x_{t 1} v_{k}+y_{t 1} u_{k}\right), \ldots,\left(-x_{t M} v_{k}+y_{t M} u_{k}\right)\right]^{T} \\
& \mathbf{c}_{5}=-j \frac{2 \pi}{\lambda}\left[\left(-x_{r 1} v_{k}+y_{r 1} u_{k}\right), \ldots,\left(-x_{r N} v_{k}+y_{r N} u_{k}\right)\right]^{T} \\
& \mathbf{C}_{6}=\left[\begin{array}{cccccc}
-\cos \theta_{k} \sin \phi_{k} & \cos \theta_{k} \cos \phi_{k} & 0 & -\cos \phi_{k} & -\sin \phi_{k} & 0 \\
-\cos \phi_{k} & -\sin \phi_{k} & 0 & \cos \theta_{k} \sin \phi_{k} & -\cos \theta_{k} \cos \phi_{k} & 0
\end{array}\right]^{T}, \\
& \mathbf{c}_{7}=\left[\cos \gamma_{k} e^{j \eta_{k}}-\sin \gamma_{k}\right]^{T} \\
& \mathbf{c}_{8}=\left[\begin{array}{ll}
j \sin \gamma_{k} e^{j \eta_{k}} & 0
\end{array}\right]^{T} \text {. }
\end{aligned}
$$

Substituting the above results into (A.3), $(i, j)$ elements of FIM $\mathbf{J}_{\boldsymbol{\theta} \boldsymbol{\theta}}$ are equal to

$$
\begin{aligned}
& \mathbf{J}_{\theta_{i} \theta_{j}} \\
& =2 L \sigma_{s_{i}}^{2} \sigma_{s_{j}}^{2} \\
& \quad \cdot \operatorname{Re}\left\{\mathbf{a}_{j}^{H} \mathbf{R}^{-1} \overline{\mathbf{b}}_{\theta_{i}} \mathbf{a}_{i}^{H} \mathbf{R}^{-1} \overline{\mathbf{b}}_{\theta_{j}}+\mathbf{a}_{j}^{H} \mathbf{R}^{-1} \overline{\mathbf{b}}_{\theta_{i}} \mathbf{a}_{i}^{H} \mathbf{R}^{-1} \overline{\mathbf{b}}_{\theta_{j}}\right\} .
\end{aligned}
$$

Define $\overline{\mathbf{A}}_{\theta}=\left[\overline{\mathbf{b}}_{\theta_{1}}, \overline{\mathbf{b}}_{\theta_{2}}, \ldots, \overline{\mathbf{b}}_{\theta_{K}}\right]$; then $\mathbf{J}_{\boldsymbol{\theta} \boldsymbol{\theta}}$ can be written as compact matrix form:

$$
\begin{aligned}
\mathbf{J}_{\boldsymbol{\theta} \boldsymbol{\theta}} & =2 \mathbf{L g g}^{T} \odot \operatorname{Re}\left\{\left(\mathbf{A}^{H} \mathbf{R}^{-1} \overline{\mathbf{A}}_{\theta}\right) \odot\left(\mathbf{A}^{H} \mathbf{R}^{-1} \overline{\mathbf{A}}_{\theta}\right)^{T}\right. \\
& \left.+\left(\mathbf{A}^{H} \mathbf{R}^{-1} \mathbf{A}\right) \odot\left(\overline{\mathbf{A}}_{\theta}^{H} \mathbf{R}^{-1} \overline{\mathbf{A}}_{\theta}\right)^{T}\right\}
\end{aligned}
$$

where $\mathbf{g}=\left[\sigma_{s_{1}}^{2}, \sigma_{s_{2}}^{2}, \ldots, \sigma_{s_{K}}^{2}\right]^{T}$. Using the identical derivation, one can obtain

$$
\begin{aligned}
& \mathbf{J}_{\boldsymbol{\theta} \psi}=\mathbf{J}_{\boldsymbol{\psi} \boldsymbol{\theta}}^{T}=2 \mathbf{L g g}^{T} \odot \operatorname{Re}\left\{\left(\mathbf{A}^{H} \mathbf{R}^{-1} \overline{\mathbf{A}}_{\phi}\right)\right. \\
&\left.\odot\left(\mathbf{A}^{H} \mathbf{R}^{-1} \overline{\mathbf{A}}_{\phi}\right)^{T}+\left(\mathbf{A}^{H} \mathbf{R}^{-1} \mathbf{A}\right) \odot\left(\overline{\mathbf{A}}_{\phi}^{H} \mathbf{R}^{-1} \overline{\mathbf{A}}_{\theta}\right)^{T}\right\},
\end{aligned}
$$

$$
\begin{aligned}
& \mathbf{J}_{\boldsymbol{\theta} \boldsymbol{\gamma}}=\mathbf{J}_{\boldsymbol{\gamma} \boldsymbol{\theta}}^{T}=2 \mathbf{L g g}^{T} \odot \operatorname{Re}\left\{\left(\mathbf{A}^{H} \mathbf{R}^{-1} \overline{\mathbf{A}}_{\gamma}\right)\right. \\
& \left.\odot\left(\mathbf{A}^{H} \mathbf{R}^{-1} \overline{\mathbf{A}}_{\theta}\right)^{T}+\left(\mathbf{A}^{H} \mathbf{R}^{-1} \mathbf{A}\right) \odot\left(\overline{\mathbf{A}}_{\gamma}^{H} \mathbf{R}^{-1} \overline{\mathbf{A}}_{\theta}\right)^{T}\right\}, \\
& \mathbf{J}_{\boldsymbol{\theta} \boldsymbol{\eta}}=\mathbf{J}_{\boldsymbol{\eta} \boldsymbol{\theta}}^{T}=2 L \mathbf{g g}^{T} \odot \operatorname{Re}\left\{\left(\mathbf{A}^{H} \mathbf{R}^{-1} \overline{\mathbf{A}}_{\eta}\right)\right. \\
& \left.\odot\left(\mathbf{A}^{H} \mathbf{R}^{-1} \overline{\mathbf{A}}_{\theta}\right)^{T}+\left(\mathbf{A}^{H} \mathbf{R}^{-1} \mathbf{A}\right) \odot\left(\overline{\mathbf{A}}_{\eta}^{H} \mathbf{R}^{-1} \overline{\mathbf{A}}_{\theta}\right)^{T}\right\}, \\
& \mathbf{J}_{\psi \psi}=2 \operatorname{Lgg}^{T} \odot \operatorname{Re}\left\{\left(\mathbf{A}^{H} \mathbf{R}^{-1} \overline{\mathbf{A}}_{\phi}\right) \odot\left(\mathbf{A}^{H} \mathbf{R}^{-1} \overline{\mathbf{A}}_{\phi}\right)^{T}\right. \\
& \left.+\left(\mathbf{A}^{H} \mathbf{R}^{-1} \mathbf{A}\right) \odot\left(\overline{\mathbf{A}}_{\phi}^{H} \mathbf{R}^{-1} \overline{\mathbf{A}}_{\phi}\right)^{T}\right\} \\
& \mathbf{J}_{\psi \gamma}=\mathbf{J}_{\gamma \psi}^{T}=2 \operatorname{Lgg}^{T} \odot \operatorname{Re}\left\{\left(\mathbf{A}^{H} \mathbf{R}^{-1} \overline{\mathbf{A}}_{\gamma}\right)\right. \\
& \left.\odot\left(\mathbf{A}^{H} \mathbf{R}^{-1} \overline{\mathbf{A}}_{\phi}\right)^{T}+\left(\mathbf{A}^{H} \mathbf{R}^{-1} \mathbf{A}\right) \odot\left(\overline{\mathbf{A}}_{\gamma}^{H} \mathbf{R}^{-1} \overline{\mathbf{A}}_{\phi}\right)^{T}\right\}, \\
& \mathbf{J}_{\psi \boldsymbol{\eta}}=\mathbf{J}_{\boldsymbol{\eta} \boldsymbol{\psi}}^{T}=2 \operatorname{Lgg}^{T} \odot \operatorname{Re}\left\{\left(\mathbf{A}^{H} \mathbf{R}^{-1} \overline{\mathbf{A}}_{\eta}\right)\right.
\end{aligned}
$$




$$
\begin{aligned}
& \left.\odot\left(\mathbf{A}^{H} \mathbf{R}^{-1} \overline{\mathbf{A}}_{\phi}\right)^{T}+\left(\mathbf{A}^{H} \mathbf{R}^{-1} \mathbf{A}\right) \odot\left(\overline{\mathbf{A}}_{\eta}^{H} \mathbf{R}^{-1} \overline{\mathbf{A}}_{\phi}\right)^{T}\right\}, \\
\mathbf{J}_{\gamma \gamma} & =2 L \mathbf{g} \mathbf{g}^{T} \odot \operatorname{Re}\left\{\left(\mathbf{A}^{H} \mathbf{R}^{-1} \overline{\mathbf{A}}_{\gamma}\right) \odot\left(\mathbf{A}^{H} \mathbf{R}^{-1} \overline{\mathbf{A}}_{\gamma}\right)^{T}\right. \\
& \left.+\left(\mathbf{A}^{H} \mathbf{R}^{-1} \mathbf{A}\right) \odot\left(\overline{\mathbf{A}}_{\gamma}^{H} \mathbf{R}^{-1} \overline{\mathbf{A}}_{\gamma}\right)^{T}\right\}, \\
\mathbf{J}_{\gamma \eta} & =\mathbf{J}_{\eta \gamma}^{T}=2 L \mathbf{g} \mathbf{g}^{T} \odot \operatorname{Re}\left\{\left(\mathbf{A}^{H} \mathbf{R}^{-1} \overline{\mathbf{A}}_{\eta}\right)\right. \\
& \left.\odot\left(\mathbf{A}^{H} \mathbf{R}^{-1} \overline{\mathbf{A}}_{\gamma}\right)^{T}+\left(\mathbf{A}^{H} \mathbf{R}^{-1} \mathbf{A}\right) \odot\left(\overline{\mathbf{A}}_{\eta}^{H} \mathbf{R}^{-1} \overline{\mathbf{A}}_{\gamma}\right)^{T}\right\}, \\
\mathbf{J}_{\eta \eta} & =2 L \mathbf{g g}^{T} \odot \operatorname{Re}\left\{\left(\mathbf{A}^{H} \mathbf{R}^{-1} \overline{\mathbf{A}}_{\eta}\right) \odot\left(\mathbf{A}^{H} \mathbf{R}^{-1} \overline{\mathbf{A}}_{\eta}\right)^{T}\right. \\
& \left.+\left(\mathbf{A}^{H} \mathbf{R}^{-1} \mathbf{A}\right) \odot\left(\overline{\mathbf{A}}_{\eta}^{H} \mathbf{R}^{-1} \overline{\mathbf{A}}_{\eta}\right)^{T}\right\},
\end{aligned}
$$

where $\overline{\mathbf{A}}_{\phi}=\left[\overline{\mathbf{b}}_{\phi_{1}}, \overline{\mathbf{b}}_{\phi_{2}}, \ldots, \overline{\mathbf{b}}_{\phi_{K}}\right], \overline{\mathbf{A}}_{\gamma}=\left[\overline{\mathbf{b}}_{\gamma_{1}}, \overline{\mathbf{b}}_{\gamma_{2}}, \ldots, \overline{\mathbf{b}}_{\gamma_{K}}\right]$, and $\overline{\mathbf{A}}_{\eta}=\left[\overline{\mathbf{b}}_{\eta_{1}}, \overline{\mathbf{b}}_{\eta_{\eta}}, \ldots, \overline{\mathbf{b}}_{\eta_{\mathrm{K}}}\right]$. Last, the CRBs are computed by the inverse of FIM J:

$$
\begin{aligned}
& \operatorname{CRB}\left(\theta_{k}\right)=\left[\mathbf{J}^{-1}\right]_{k, k}, \\
& \operatorname{CRB}\left(\phi_{k}\right)=\left[\mathbf{J}^{-1}\right]_{K+k, K+k}, \\
& \operatorname{CRB}\left(\gamma_{k}\right)=\left[\mathbf{J}^{-1}\right]_{2 K+k, 2 K+k}, \\
& \operatorname{CRB}\left(\eta_{k}\right)=\left[\mathbf{J}^{-1}\right]_{3 K+k, 3 K+k},
\end{aligned}
$$

\section{Competing Interests}

The authors declare that there are no competing interests regarding the publication of this paper.

\section{Acknowledgments}

This work was supported by National Natural Science Foundation of China under Grant no. 61501504, China Postdoctoral Science Foundation under Grant no. 2015M581097, and Natural Science Basic Research Plan in Shaanxi Province of China under Grant no. 2016JQ6020.

\section{References}

[1] E. Fishler, A. Haimovich, R. Blum, D. Chizhik, L. Cimini, and R. Valenzuela, "MIMO radar: an idea whose time has come," in Proceedings of the IEEE Radar Conference, pp. 71-78, Honolulu, Hawaii, USA, April 2004.

[2] I. Bekkerman and J. Tabrikian, "Target detection and localization using MIMO radars and sonars," IEEE Transactions on Signal Processing, vol. 54, no. 10, pp. 3873-3883, 2006.

[3] J. Li, P. Stoica, L. Xu, and W. Roberts, "On parameter identifiability of MIMO radar," IEEE Signal Processing Letters, vol. 14, no. 12, pp. 968-971, 2007.
[4] L. Xu, J. Li, and P. Stoica, "Target detection and parameter estimation for MIMO radar systems," IEEE Transactions on Aerospace and Electronic Systems, vol. 44, no. 3, pp. 927-939, 2008.

[5] C. Duofang, C. Baixiao, and Q. Guodong, "Angle estimation using ESPRIT in MIMO radar," Electronics Letters, vol. 44, no. 12, pp. 770-771, 2008.

[6] G. Zheng, B. Chen, and M. Yang, "Unitary ESPRIT algorithm for bistatic MIMO radar," Electronics Letters, vol. 48, no. 3, pp. 179-181, 2012.

[7] X. Zhang, L. Xu, L. Xu, and D. Xu, "Direction of departure (DOD) and direction of arrival (DOA) estimation in MIMO radar with reduced-dimension MUSIC," IEEE Communications Letters, vol. 14, no. 12, pp. 1161-1163, 2010.

[8] B. Tang, J. Tang, Y. Zhang, and Z. Zheng, "Maximum likelihood estimation of DOD and DOA for bistatic MIMO radar," Signal Processing, vol. 93, no. 5, pp. 1349-1357, 2013.

[9] J. Li and X. Zhang, "Joint angles and mutual coupling estimation algorithm for bistatic MIMO radar," International Journal of Antennas and Propagation, vol. 2012, Article ID 921878, 8 pages, 2012.

[10] C. Yunhe, Z. Zijing, W. Shenghua, and D. Fengzhou, "Direction finding for bistatic MIMO radar with uniform circular array," International Journal of Antennas and Propagation, vol. 2013, Article ID 674878, 6 pages, 2013.

[11] H. Jiang, D.-F. Wang, and C. Liu, "Joint parameter estimation of DOD/DOA/polarization for bistatic MIMO radar," The Journal of China Universities of Posts and Telecommunications, vol. 17, no. 5, pp. 32-37, 2010.

[12] M. L. Bencheikh and Y. Wang, "Joint DOD-DOA estimation using combined ESPRIT-MUSIC approach in MIMO radar," Electronics Letters, vol. 46, no. 15, pp. 1081-1083, 2010.

[13] M. L. Bencheikh and Y. Wang, "Combined ESPRITRootMUSIC for DOA-DOD estimation in polarimetric bistatic MIMO radar," Progress In Electromagnetics Research Letters, vol. 22, pp. 109-117, 2011.

[14] H. Jiang, Y. Zhang, J. Li, and H. Cui, "A PARAFAC-based algorithm for multidimensional parameter estimation in polarimetric bistatic MIMO radar Emerging radar techniques," EURASIP Journal on Advances in Signal Processing, vol. 2013, article 133, 2013.

[15] C. Gu, J. He, H. Li, and X. Zhu, "Target localization using MIMO electromagnetic vector array systems," Signal Processing, vol. 93, no. 7, pp. 2103-2107, 2013.

[16] A. Nehorai and E. Paldi, "Vector-sensor array processing for electromagnetic source localization," IEEE Transactions on Signal Processing, vol. 42, no. 2, pp. 376-398, 1994.

[17] K. T. Wong and M. D. Zoltowski, "Closed-form direction finding and polarization estimation with arbitrarily spaced electromagnetic vector-sensors at unknown locations," IEEE Transactions on Antennas and Propagation, vol. 48, no. 5, pp. 671-681, 2000.

[18] G. Zheng, B. Wu, Y. Ma, and B. Chen, "Direction of arrival estimation with a sparse uniform array of orthogonally oriented and spatially separated dipole-triads," IET Radar, Sonar \& Navigation, vol. 8, no. 8, pp. 885-894, 2014.

[19] G. Zheng, B. Chen, and M. Yang, "Joint 2D-DOA and polarization estimation using a sparse uniform array of spatially noncollocating electromagnetic vector sensors," Scientia Sinica, vol. 44, no. 9, pp. 1171-1192, 2014.

[20] H. L. Trees, Detection, Estimation, and Modulation Theory, Part IV: Optimum Array Processing, Wiley Press, New York, NY, USA, 2002. 


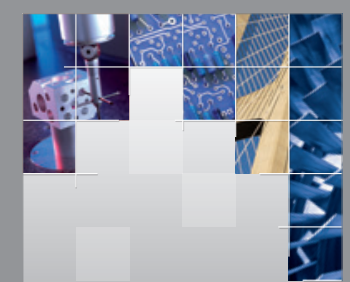

\section{Enfincering}
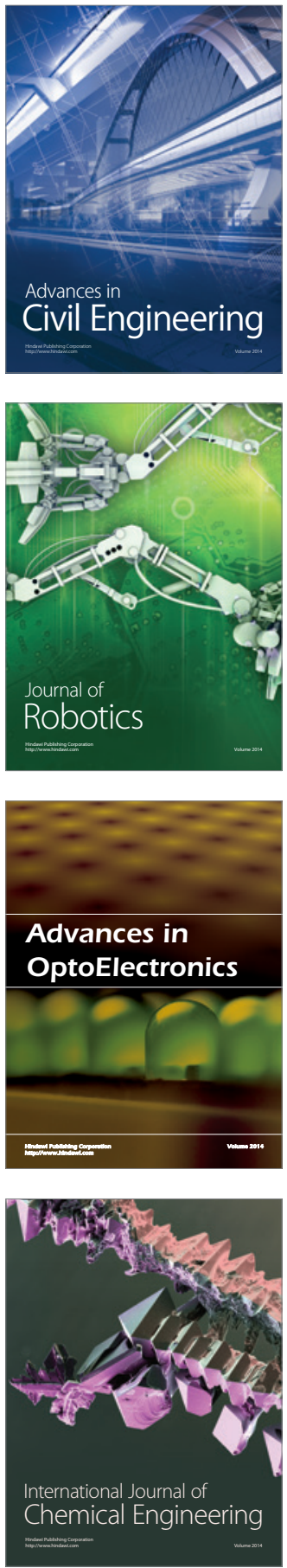

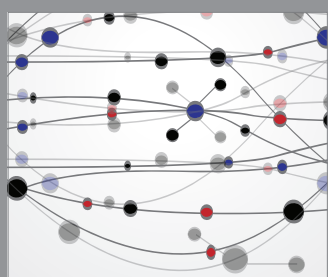

The Scientific World Journal

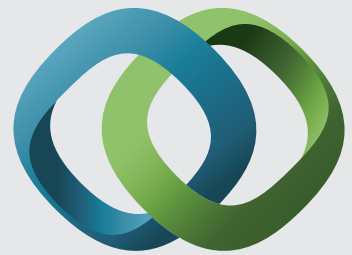

\section{Hindawi}

Submit your manuscripts at

http://www.hindawi.com
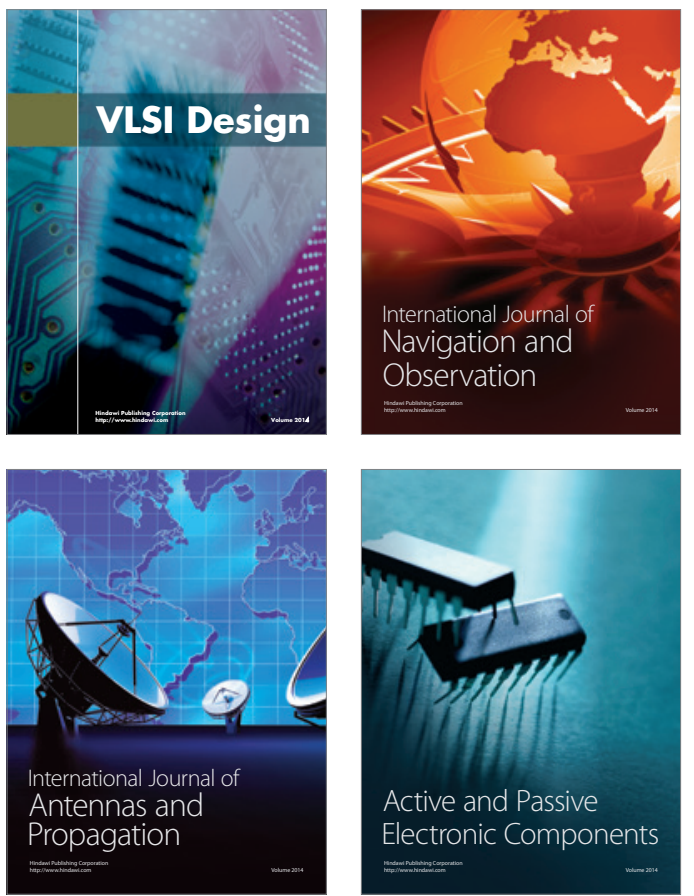
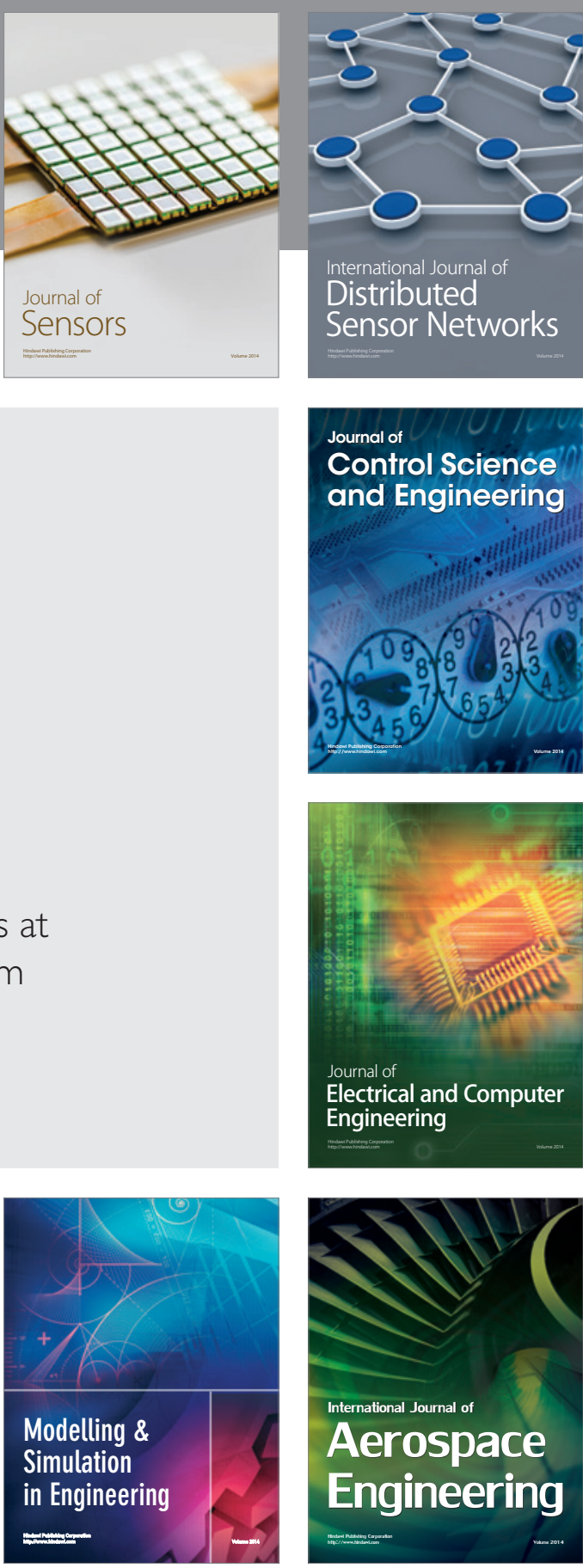

International Journal of

Distributed

Sensor Networks

Journal of

Control Science

and Engineering
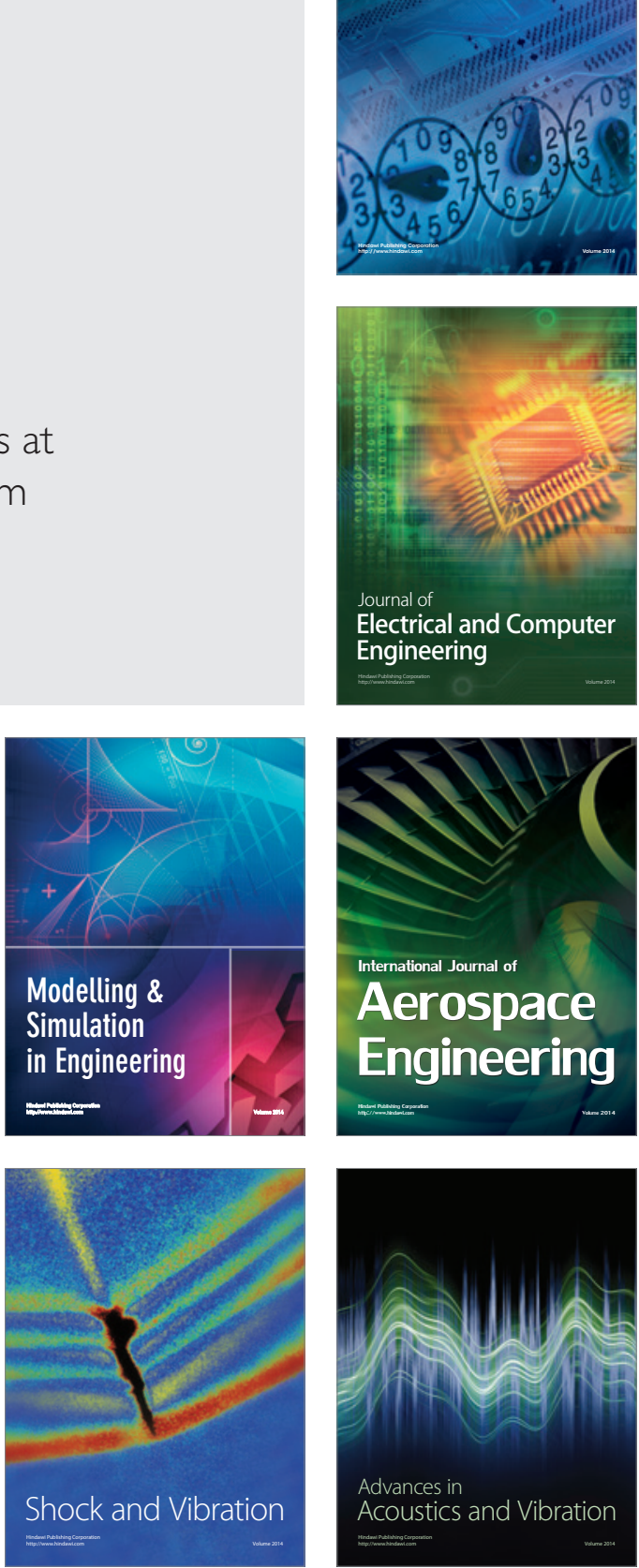